\title{
THREE-PHOTON EXCITATION IN FLUORESCENCE MicRoscopy
}

\author{
Stefan W. Hell, ${ }^{\dagger}$ Karsten Bahlmann, ${ }^{\dagger *}$ Martin Schrader, ${ }^{\dagger}$ Aleksi Soini, ${ }^{\dagger}$ Henryk Malak, \\ Ignacy Gryczynski, ${ }^{\star}$ and Joseph R. Lakowicz ${ }^{\ddagger}$ \\ ${ }^{\dagger}$ University of Turku, Dept. of Medical Physics and Chemistry, P.O. Box 123, FIN-20521 Turku, \\ Finland; 'Universität Heidelberg, Institut für Angewandte Physik, Albert-Überle-Strasse 3-5, D- \\ 69120, Heidelberg, Germany; University of Maryland School of Medicine, Center for \\ Fluorescence Spectroscopy, Department of Biological Chemistry, Baltimore, MD \\ (Paper JBO-060C received Nov. 29, 1995; revised manuscript received Dec. 11, 1995; accepted for publication Dec. 12, 1995)
}

\begin{abstract}
We show experiments proving the feasibility of scanning fluorescence microscopy by three-photon excitation. Three-photon excitation fluorescence axial images are shown of polystyrene beads stained with the fluorophore 2,5-bis(4-biphenyl)oxazole (BBO). Three-photon excitation is performed at an excitation wavelength of $900 \mathrm{~nm}$ and with pulses of $130 \mathrm{fs}$ duration provided by a mode-locked titanium-sapphire laser. Fluorescence is collected between 350 and $450 \mathrm{~nm}$. The fluorescence image signal features a third-order dependence on the excitation power, also providing intrinsic 3-D imaging. The resolution of a three-photon excitation microscope is increased over that of a comparable two-photon excitation microscope.
\end{abstract}

Key Words Three-photon excitation, fluorescence microscopy, resolution.

Recently, two-photon excitation has evolved as a powerful way of performing three-dimensional fluorescence scanning microscopy. ${ }^{1}$ In a two-photon excitation microscope, the fluorophore is excited by the simultaneous absorption of two photons of about twice the absorption wavelength, thus allowing the excitation of UV fluorophores with visible or near-infrared light. At present, mode-locked Ti:sapphire lasers are the most popular light sources for two-photon imaging. Ti:sapphire lasers provide intense subpicosecond pulses at a high repetition rate from about $690 \mathrm{~nm}$ to $1000 \mathrm{~nm}$. With a Ti:sapphire laser, two-photon excitation is restricted to dyes with single photon absorbance of 345 to 500 $\mathrm{nm}$. Since many fluorochromes have their maximum absorbance between 200 and $350 \mathrm{~nm}$, twophoton excitation of such dyes is somewhat hampered by the lack of suitable light sources. A possible approach to this problem is the use of continuous-wave lasers operating at a higher average power. ${ }^{2}$ A promising alternative is the additional absorption of a third photon for bridging the energy gap between the ground and the excited state. In principle, one would expect three-photon excitation to be a rather unlikely event, hardly capable of producing any fluorescence signal, but recent research has shown that with certain compounds, significant fluorescence can be generated by the simultaneous absorption of three photons. ${ }^{3-6}$ These results encouraged us to investigate the feasibility of scanning fluorescence microscopy by three-photon excitation. Here, we report about

Address all correspondence to Stefan W. Hell. E-mail: stefan.hell@utu.fi what is, to our knowledge, the first evidence of microscopic fluorescence imaging by three-photon excitation.

The following experiments were carried out with a stage scanning fluorescence microscope employing a mode-locked Ti:sapphire laser for illumination (Mira 900, Coherent Inc.). The Ti:sapphire laser provided a stream of infrared pulses of $130 \mathrm{fs}$ duration at a rate of $76 \mathrm{MHz}$. The pulses were deflected by a dichroic mirror and focused into the sample by an oil immersion lens of high numerical aperture. The same lens was used to collect the light from the sample. The dichroic mirror was transparent for wavelengths shorter than $680 \mathrm{~nm}$. The light coming from the sample was filtered by two dichroic shortwave pass filters transmitting below $450 \mathrm{~nm}$ and $600 \mathrm{~nm}$, respectively. In addition, a bandpass filter transmitting between 300 and $450 \mathrm{~nm}$ and a 4-mm thick stack of BG39 (Schott, Mainz, Germany) was placed in front of the detector. The stack of colored glass was specified to suppress the laser light by 20 orders of magnitude. The combination of filters ensured that the maximum transmission was around 410 to $420 \mathrm{~nm}$, which is more than twice as short as the excitation wavelength. The photomultiplier was blue sensitive and operating in the photon counting mode. The piezoelectric scanning stage (Physik Instrumente, Waldbronn, Germany) positioned the sample with an accuracy of 10-20 nm in three dimensions. The entrance pupil of the lens was illuminated with an expanded beam, 2-3 times larger than the entrance pupil of the lens. Taking into ac-

1083-3668/96/\$6.00 @ 1996 SPIE 


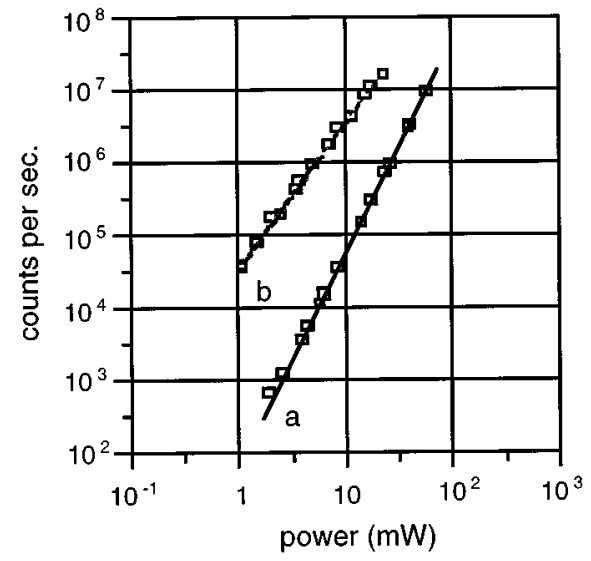

Fig. 1 Fluorescence yield as a function of the time-averaged excitation power entering the objective lens for (a) BBO in tolvene, and (b) rhodamine $6 \mathrm{G}$ in immersion oil recorded with an excitation wavelength of $900 \mathrm{~nm}$.

count the repetition rate, the pulse length, and a focal spot diameter of about $400 \mathrm{~nm}$, we can calculate that $1 \mathrm{~mW}$ of average illumination power generates a focal peak intensity of about $50 \mathrm{GW} / \mathrm{cm}^{2}$.

Figure 1(a) shows the fluorescence signal as it is obtained by focusing into a concentrated solution of BBO in toluene. BBO $(99 \%$ scintillation grade, Aldrich) has its maximum absorbance between 280 and $380 \mathrm{~nm}$ and emits primarily between 370 and 480 $\mathrm{nm}$. This compound was chosen because we know that it yields considerable three-photon fluorescence when using excitation wavelengths above 820 $\mathrm{nm} .{ }^{4}$ The illumination power entering the objective lens was varied by neutral optical density filters. The solution was mounted between two cover slips, forming a 10 to $15-\mu \mathrm{m}$ thick layer. The wavelength of the illumination light was $900 \mathrm{~nm}$. The numerical aperture of the lens (Leica Planapochromat, 100x) was 1.4. The maximum average illumination power was about $60 \mathrm{~mW}$. The slope in Fig. 1(a) is $2.87 \pm 0.2$, indicating that the generation of the fluorescence signal is governed by a third-order process. We also prepared a layer of rhodamine 6G (Kodak, Rochester, NY) dissolved in immersion oil. When measuring the fluorescence of rhodamine 6G, the bandpass filter and the 450-nm short-wave pass filter was removed. The fluorescence of rhodamine 6G [Figure 1(b)] showed a quadratic dependence on excitation intensity with a slope of $2.02 \pm 0.2$. At a wavelength of $900 \mathrm{~nm}$, the fluorescence of rhodamine 6G is generated by the simultaneous absorption of two photons whereas that of $\mathrm{BBO}$ is primarily due to the absorption of three photons.

Fluorescence layers enable a direct comparison of the axial resolution of a two- and three-photon excitation microscope by axially scanning the boundary between the cover slip and the solution. The opening of the detector was $4 \mathrm{~mm}$, which was

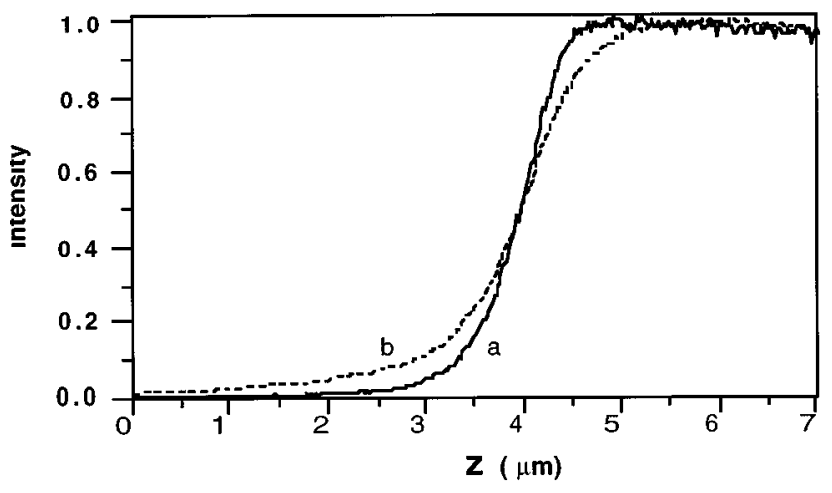

Fig. 2 Signal obtained when moving the focus from the cover glass into the (a) $\mathrm{BBO} /$ toluene and (b) rhodamine 6G/immersion oil layer as a measure of the axial resolution and discrimination capability. The excitation wavelength is $900 \mathrm{~nm}$. No confocal spatial filtering is used. The signal-to-noise ratio is enhanced by averaging over 10 consecutive recordings. The steeper signal in (a) proves the higher axial resolution of three-photon excitation microscopy.

about 50 times larger than the backprojected excitation focus. An opening of this size excludes any potential discrimination through confocal spatial filtering. Figure 2 depicts the axial edge responses for the (a) BBO and (b) rhodamine 6G layer. The slope of the axial response is a good measure of the axial resolution. Moreover, a comparison of the two edges reveals the axial resolution performance in both cases. Figure 2 clearly shows that with threephoton excitation the axial edge is steeper. A useful criterion for the axial resolution is the axial distance between the 20 and $80 \%$ threshold value of the edge, $\Delta Z_{20-80 \%}$. The two-photon fluorescence axial edge has a $\Delta Z_{20-80 \%}=1020 \pm 80 \mathrm{~nm}$, whereas for three-photon excitation we obtained $\Delta Z_{20-80 \%}$ $=680 \pm 80 \mathrm{~nm}$.

As a next step, we stained polystyrene beads 2 $\mu \mathrm{m}$ and $20 \mu \mathrm{m}$ in diameter with BBO, and imaged the beads with the scanning microscope. The preparation of the $20-\mu \mathrm{m}$ beads was more difficult because the concentration was not uniformly distributed and was considerably stronger at the outer region of the beads. The beads were dried on a microscope slide, mounted in glycerol and covered by a cover glass. Small crystals of BBO were also dispersed in the sample, so that we could image crystals as well as beads.

Figure 3(a) shows an axial image of a microcrystal imaged with an oil immersion lens of numerical aperture 1.3 (Zeiss Plan Neofluar, 100×). The size of the image was $3 \times 4 \mu \mathrm{m}$. The pixel size was $20 \mathrm{~nm}$ and the pixel dwell time $2 \mathrm{~ms}$. The average power (continuous wave equivalent) entering the objective lens was $3.5 \mathrm{~mW}$. The elongation of the microcrystal image is mostly due to the elongation of a single lens focus along the optical axis. ${ }^{7}$ The crystal is axially resolved, thus showing the higher order excita- 
a)

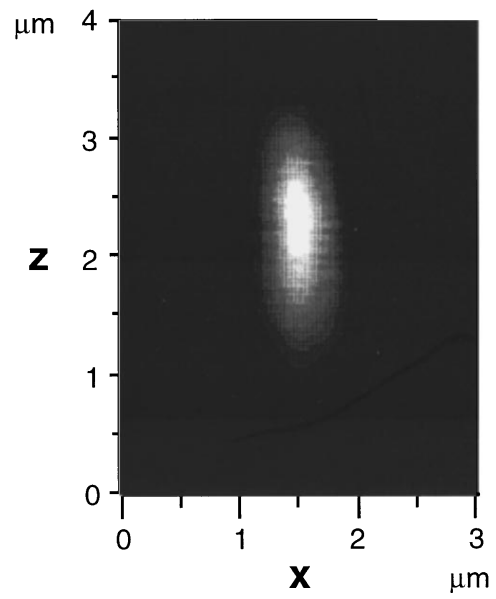

b)

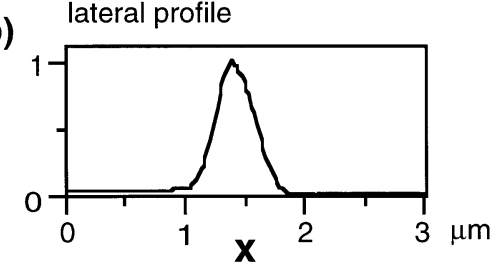

c)

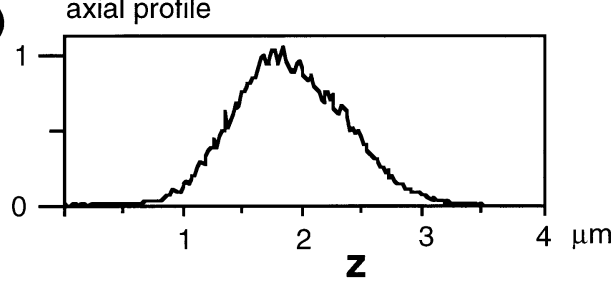

Fig. 3 (a) Axial image (XZ-section) of a microcrystal of $\mathrm{BBO}$; (b) lateral profile; and (c) axial profile.

tion of the fluorescence signal. Figure 3(b) shows the lateral profile from the image of Figure 3(a), as found at the coordinate $z=2.2 \mu \mathrm{m}$. Figure 3(c) shows the axial profile at the coordinate $x=1.3 \mu \mathrm{m}$. The full-width-at-half-maximum (FWHM) of the lateral profile is $380 \pm 20 \mathrm{~nm}$, whereas the axial FWHM is $1080 \pm 50 \mathrm{~nm}$. An ideal, aberration-free, three-photon excitation microscope operating at a wavelength of $900 \mathrm{~nm}$ and a numerical aperture of 1.35 has a theoretically predicted lateral and axial resolution (FWHM) of about $200 \mathrm{~nm}$ and $500 \mathrm{~nm}$, respectively. Some of the difference in the lateral and axial profiles in Figures 3(b) and 3(c) is because of the limited performance of the objective lenses at $900 \mathrm{~nm}$, but mostly because the microcrystal was not "pointlike." ${ }^{7}$ Figure 4(a) displays an axial image of a cluster of $2 \mu \mathrm{m}$ polystyrene beads stained with $\mathrm{BBO}$. The average power was $2.2 \mathrm{~mW}$. The shape of the beads is clearly visible. The background signal of unstained beads was about three orders of magnitude lower than the signal from BBO-stained beads. We also recorded axial and lateral images of BBO-stained polystyrene beads of $20 \mu \mathrm{m}$ diameter. All images revealed an intrinsic axial discrimination.

In the first experimental report on two-photon fluorescence microscopy, ${ }^{1}$ the nonlinear dependence of the fluorescence signal on the excitation power was demonstrated by proving axial discrimination without a confocal pinhole. However, this approach does not allow for distinguishing threefrom two-photon excitation, or from any other multiphoton excitation. The ultimate test of the threephoton nature requires a measurement of the fluorescence signal as a function of the excitation power. We have measured the intensity dependence of the fluorescence for $20-\mu \mathrm{m}$ and $2-\mu \mathrm{m}$ stained polystyrene beads. We determined this dependence by recording a series of 14 axial images of $2-\mu \mathrm{m}$ bead clusters taken at the same site of the sample but with different excitation power. Figure 4(b) shows the mean fluorescence signal of each axial image as a function of the excitation power at which the image was taken. The double-logarithmic plot reveals a slope of $2.8 \pm 0.2$, providing a further piece of evidence that the images were generated by the simultaneous absorption of three photons.

In conclusion, our experiments show the feasibility of fluorescence imaging by three-photon excitation.* We have exemplified this by imaging microscopic structures stained with the fluorophore BBO. At an excitation wavelength of $900 \mathrm{~nm}$, the axial resolution of the three-photon excitation microscope was determined as $680 \pm 80 \mathrm{~nm}$, which is significantly better than a comparable two-photon excitation microscope. All the optical components were of the standard type and not corrected for the $900-\mathrm{nm}$ or 350 to $450-\mathrm{nm}$ wavelength range. We believe that dedicated correction of chromatic aberrations holds promise for further improvement in resolution both for the three- and two-photon microscope operating around $900 \mathrm{~nm}$. Since the excitation light is not visible, careful alignment of the beam is important to achieve optimal resolution. However, due to the third-order intensity behavior, the axial discrimination of a three-photon microscope is stronger than in a comparable two-photon microscope, as we have demonstrated in Figure 2.

Three-photon excited fluorescence of $\mathrm{BBO}$ is about two orders of magnitude lower than comparable two-photon excited fluorescence of the same compound. ${ }^{4}$ Currently, an average power of up to 5 to $15 \mathrm{~mW}$ (100 fs pulses, excitation around $730-780$ $\mathrm{nm}, 80 \mathrm{MHz}$ repetition rate) is considered to be tolerable in a biological specimen. ${ }^{8}$ The applicability to biological imaging will strongly depend on the tol-

*Part of the results shown here were presented at a seminar of the Faculty of Applied Physics, University of Twente, Enschede, The Netherlands, September 20, 1995. 
a)

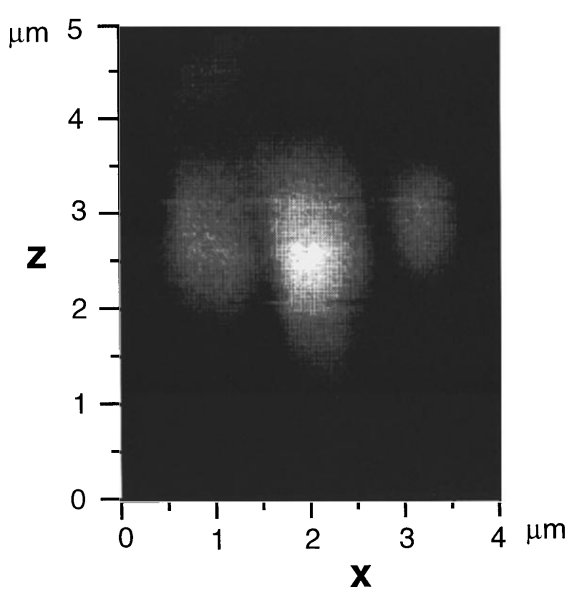

b)

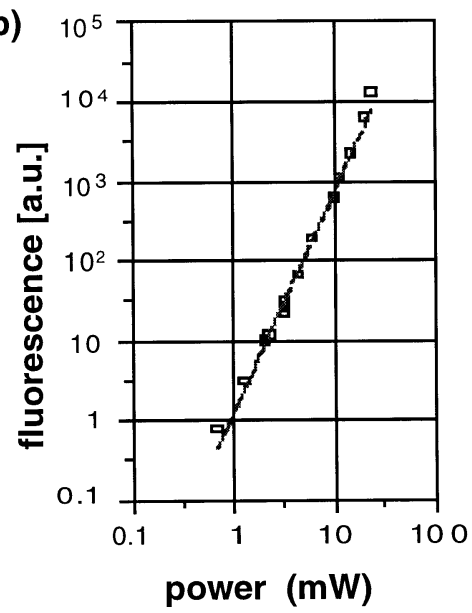

Fig. 4 (a) BBO-stained polystyrene beads imaged with three-photon excitation. (b) The dependence of the mean brightness of the image on the average excitation power.

erances of the specimens to high intensities and powers in the 850 to $1000-\mathrm{nm}$ range. In any case, information about the presence or absence of the fluorophore in a given part of the specimen will be retrievable with a three-photon fluorescence microscope. The fact that other compounds, such as the calcium marker Indo-1, and proteins are reported to exhibit three-photon fluorescence ${ }^{3,6}$ leads us to conclude that three-photon excitation microscopy is likely to evolve as a new kind of fluorescence imaging with considerable potential for application.

\section{REFERENCES}

1. W. Denk, J. H. Strickler, and W. W. Webb, "Two-photon fluorescence scanning microscopy," Science 248, 73-75 (1990).

2. P. E. Hänninen, E. Soini, and S. W. Hell, "Continuous wave two-photon excitation microscopy," J. Microscop. 176, Pt 3, 222-225 (1994).
3. I. Gryczynski, H. Szmaczinski, and J. R. Lakowicz, "On the possibility of calcium imaging using Indo-1 with threephoton excitation," Photochem. Photobiol. 62, 804-808 (1995).

4. I. Gryczynski, H. Malak, S. W. Hell, and J. R. Lakowicz, "Three-photon excitation of 2,5-bis(4-biphenyl)oxazole," submitted.

5. G. S. He, J. D. Bhalkawar, P. N. Prasad, and B. A. Rhinhard, "Three-photon absorption-induced fluorescence and optical limiting effects in an organic compound," Opt. Lett. 20, No. 14, 1524-1526 (1995).

6. I. Gryczynski, H. Malak, and J. R. Lakowicz, "Three-photon excitation of tryptophan derivative using a fs Ti:sapphire laser," Biospectroscopy (1996), in press.

7. S. W. Hell, P. E. Hänninen, A. Kuusisto, M. Schrader, and E. Soini, "Annular aperture two-photon excitation microscopy," Opt. Commun. 117, 20-24 (1995).

8. W. Denk, D. W. Piston, and W. W. Webb, "Two-photon molecular excitation in laser scanning microscopy," in Handbook of Biological Confocal Microscopy, J. Pawley, Ed., 2nd ed., pp. 445-457, Plenum Press, New York (1995). 